\title{
QR Code Steganography for Multiple Image and Text Hiding using Improved RSA-3DWT Algorithm
}

\author{
Shweta Sharma ${ }^{1}$ and Prof. Vikas Sejwar ${ }^{2}$ \\ ${ }^{1,2}$ CSE/IT Department MITS, Gwalior, India \\ ${ }^{1}$ shwetasharma675@gmail.com, ${ }^{2}$ vikassejwar@mitsgwalior.in
}

\begin{abstract}
In this research, we presented another implanting calculation for QR Code Image Steganography and Text Hiding, which is based on 3-discrete wavelet transform (DWT) and improved RSA algorithm. Firstly, enter the text message and choose four random numbers for RSA encryption. After that, take four pictures: one is cover image and another is secret image. In the process of embedding, we divide RGB image into three planes: Red, Green and Blue. In this work, embed multiple color secret images into a single cover image for providing security. At last, encrypted text message is concealed into an embedded picture using Least significant bit (LSB). We evaluate performance on the basis of the peak signal noise ratio (PSNR) and structural similarity matrix (SSIM) with different varying weights. In this proposed, compared this algorithm to 1-DWT. In the extraction process, recover three secret image from stego image and decrypt text message.In this experiment, improved PSNR value as compared to previous paper. The experimental outcomes will be reached upto $84 \%$. In our method, DWT is applied to decompose the cover image and produce the low and high frequency coefficients to take benefits of robustness and security goods.
\end{abstract}

Keywords: DWT; PSNR; RSA, SSIMb(structural similarity matrix); Image Steganography

\section{Introduction}

The rapid progress within the digital technology, image processing tools and conversation revolution by means of the web has made the reproduction of digitally created artifacts easy and inside attain very quite simply. Image processing is a procedure to convert a picture into digital form and contribute in a few operations on it, with an analysis to obtain a superior picture or to extract some valuable prospective from it. It's a form of signal dispensation the place input is an image, like video body or picture and output would even be an image or characteristics involving that image. In image Processing approach pix are handled as two dimensional indicators at the same time applying already set sign processing ways to them[1].

Steganography is the science and art of thrashing bythe manner of embedding messages with additional apparently harmless messages. Steganography means - protected writing\| in Greek. Because the rationale of steganography is to divet the presence of a message and to create a covert channel, it may be visible on the grounds that that the complement of cryptography, whose intention is to cover the content data of a message. An extra form of expertise hiding is digital watermarking, which is the method that embeds capabilities often called a watermark, tag or label correct right into multimedia item such that watermarks will also be recognized or extracted afterward to create a statement concerning the thing. The item may be a picture, text, sound file or video. A well-known design of steganography is Simmons' Prisoners' very essential drawback [2]. An assumption will also be made situated on this mannequin is that if both the sender and receiver share some normal secret knowledge then the corresponding 
steganography protocol is often called then the key key steganography the place as pure steganography manner that there is none prior understanding shared by means of sender and receiver. If the general public key of the receiver is legendary to the sender, the steganographic protocol is known as public key steganography [3], [4] and [5].For a more thorough abilities of steganography methodology the reader is advised to see [6-7].Some Steganographic mannequin with excessive security aspects. Close to all digital file formats can be used for steganography, but the image and audio documents are extra compatible on account that of their excessive measure of redundancy [8].

In this age of the digital world, with the progress of technology and continuous growth in digital data, there is a vital need of optimization of data and information presently in the digital world. The authenticity of data is the trickiest issue in management of data in the internet database. In this, we mainly consider the authenticity of security passwords, phonenumbers or security information. This process also applied to steganogrphy technique to hide the secret images, route maps.

Furthermore, there is no second certification on human eye verification of documents. Keeping this problem in mind, wehave introduced a new digital documentation system using QR codes.QR Code is a form of 2-D matrix barcode, that is more admired than 1-D barcodes due to its great capability of digital information and it can be legible in every mobile phones. In our original paper, we keep the necessary data for example signature, passwords within QR Code. But, whole data saved within QR Code, are encrypted, then printed on the paper.[9]

\section{RSA Methodology}

We use the same key in the TTJSA algorithm in the decryption and encryption side. As a result of this reason the data can be modified by the other user. To resolve this problem we can use the RSA algorithm. In this algorithm we use different key on both sides. On the encryption side, we utilize the private key in the equal manner in decryption side we utilize a public key.

The RSA cryptosystem is one of the well-known security algorithm. There are mainly three steps in RSA algorithm:

Encryption, decryption and key generation procedure.

\section{Key Generation:}

1. Take the two numbers $\mathrm{p}, \mathrm{q}$ and $\mathrm{p} \neq \mathrm{q}$

2. Compute $\mathrm{n}=\mathrm{p} \times \mathrm{q}$.

3. $\varphi(n)$ computed as $\varphi(n)=(p-1) \times(q-1)$.

4. Take integer e whose $\operatorname{gcd}(\varphi(\mathrm{n}), \mathrm{e})=1,1<\mathrm{e}<\mathrm{n}$.

5. Consider such that Ed-1 is accurately dividable by $\varphi(n)$.

6. Private key $(\mathrm{d}, \mathrm{n})$.

7. Public key $(e, n)$.

\section{Encryption process}

Plain text --- $\mathrm{m}$

Ciphertext $\mathrm{C}=\left(\left(\mathrm{m}^{\wedge} \mathrm{e}\right) \bmod \mathrm{n}\right)$.

Decryption process

Ciphertext --- C

Decipher text $\mathrm{D}=\left(\left(\mathrm{C}^{\wedge} \mathrm{d}\right) \bmod \mathrm{n}\right)$.

Such as, consider $\mathrm{p}=5, \mathrm{q}=3$. So, $\varphi(\mathrm{n})=(5-1)(3-1)=8$.

so, $\mathrm{e}=11, \mathrm{~d}=3$.

Plain text --- 2.

Ciphertext $\mathrm{C}=(211 \bmod 15)=2048 \bmod 15=8$.

Decipher text $\mathrm{D}=(83 \bmod 15)=512 \bmod 15=2$. 
$\mathrm{D}=\mathrm{m}$.

\section{Discrete Wavelet Transforms}

Wavelets likewise speaking to indicators in a structure closely resembling sines and cosines while tending to the issue with Fourier investigation. DWT is a change focused around recurrence space. In DWT the flow of the reappearance is altered in every one phase of DWT, where L is Low recurrence, $\mathrm{H}$ is High recurrence and subscript behind them is the number of convert layers [10]. Sub diagram LL is a low recurrence band the additional level resolve rough presumption of the foremost feature, HL vertical high recurrence band, $\mathrm{LH}$ a flat high recurrence band, $\mathrm{HH}$ an inclining high recurrence band. The most visible data in the sign represent high amplitudes and the less prominent data represent low amplitudes in DWT. Information clamping might be accomplished via tossing these low amplitudes. The wavelet converts empowers high packing degrees with great nature of recreation Wavelet convert escapable of giving the time and recurrence data all the while, Therefore giving a time frequency illustration of the signal. DWT is conventional to every proper model component of the HVS (Human Visual System) as contrasted with the DCT or FFT. This allows to utilize great vitality watermarks as a component of districts that the HVS is recognized to be small delicate to including watermarks in these areas enhances the capability of watermark, additional effect on image quality. Uncertainly it is, no uncertainty found that addition in the LL section of the DWT produce very dominant adjacent to different types of attacks.[11]

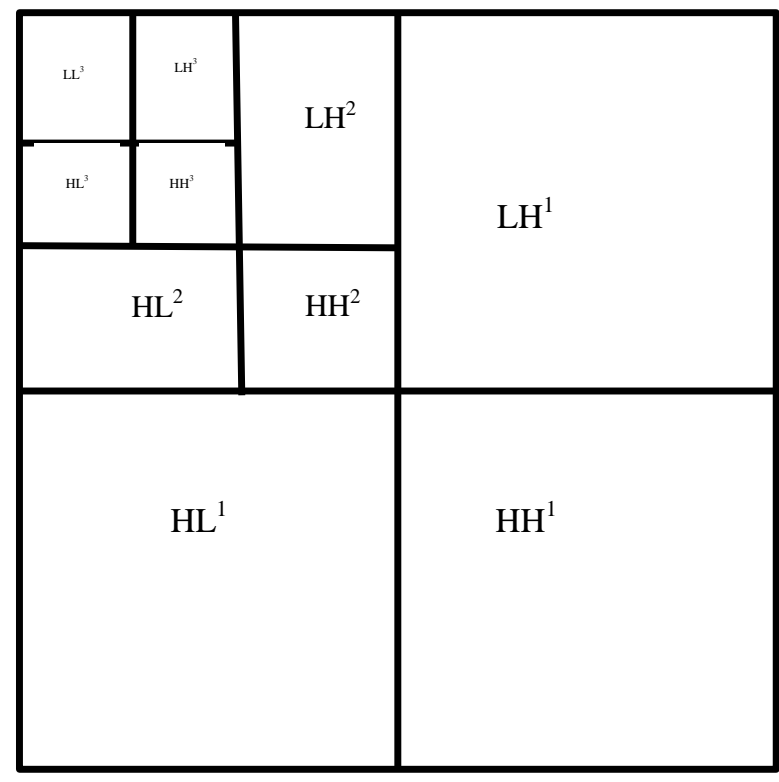

$$
\begin{aligned}
& \text { 1,2,3------Decomposition } \\
& \text { levels } \\
& \text { H----------high frequency } \\
& \text { bands } \\
& \text { L---------low frequency }
\end{aligned}
$$
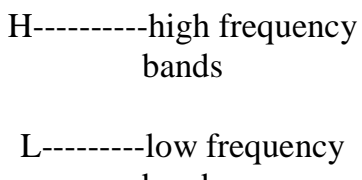

Figure 1. DWT Filter up to 3-Level

One of the major power of WT compared to the DFT and DCT is its resemblance with HVS which permits the watermark to be embedded in the area that the HVS is recognized as less perceptive to, for example the high resolution detail bands HL, HH and LH [12]. Embedding watermarks in these areas let us to enhance the strenght of our watermark, at small to no other impact on picture quality.

\section{Literature Survey}

Vijay Kumar and Dinesh Kumar explained DWT based image steganography approach.In this paper, The blocks of approximation coefficients of cover image are 
subtracted from approximation coefficient of secret image. The differences of these coefficients are called error blocks. The replacement of an error block is being done with the best matched $\mathrm{CH}$ block. In this method, besides using $\mathrm{CH}$ block, we have done experimentation using $\mathrm{CV}$ and $\mathrm{CD}$ blocks also to see the effect on PSNR of the steganoimage[12].

In this paper, We enhance our area of research by encrypting image data using RSA and use a color image for embedding process. In this combined process of Steganography, Digital Watermarking and Cryptography make use of Discrete cosine transform(DCT), Discrete wavelet transform(DWT), Singular value decomposition(SVD) and RSA algorithm which furnish safety of the images and as well as authenticity of the image author in the internet atmosphere. The proposed approach for protection of image for transmission could be very beneficial in 738 web the place large person sends images by way of mail, social networking sites. It has a crucial role in securing with general applications. This would also be beneficial in space technology where satellite sends images of planets preventing it from going in wrong hands. This would be beneficial to nation for overall security. [1]

Amitava Nag et. al., [13] presents a novel technique for Image steganography based on DWT. To start with $2 \mathrm{~d}$ DWT is executed on a gray stage cover picture of $\mathrm{M} \times \mathrm{N}$ dimension and Huffman encoding is taken out on the covert messages former than embedding. Then all bits of Huffman code of covert message are embedded during the great frequency coefficients consequences from DWT. Image high-quality is to be elevated with the aid of keeping the wavelet coefficients within the low frequency subband. The experimental outcome suggests that the algorithm has a high ability and an excellent invisibility. PSNROf canopy snapshot with stego-photograph shows the less complicated outcome in evaluation with specific current steganography approaches. In this paper, the predominant value is given on the secrecy as well as the privateness of information.

Raj J. Jaiswal, et. al., [14]The very general public key algorithm is an RSA cryptosystem utilized for encryption and decryption. It is the foremost public key algorithm that gives transfer protection and data saving above the network. In this there is less protection and time computation is still prolonged. This paper advised a novel algorithm idea to show the changed form of RSA algorithm so as to boost up the execution of RSA algorithm throughout the data swap on the network. This combined the architectural aim and improved RSA algorithm throughout use of third prime digit so as to build a modulus that is not simply decomposable by intruders. A database system is used to store the key parameters of RSA cryptosystem before it starts the algorithm. Lastly, we evaluate projected RSA technique with the unique RSA scheme by some theoretical features. Proportional outputs offer enhanced security with projected algorithm.

In paper[15], PrabakaranGanesan et. al., Proposed a high cozy steganography scheme hiding a $256 \times 256$ dimension gray secret image right into a $512 \times 512$ measurement grey secret image with an extraordinary mixture of Discrete Wavelet transform and Integer Wavelet transform (IWT). Pixel valued at Adjustment is first carried out on coverimage. The secret image values are scrambled by way of using Arnold develop into. The DWT /IWT is applied on each the quilt and scrambled secret image. Blending method is utilized to both images and compute I DWT/IWT on the identical to get the stego image. The extraction model is the reverse procedure of the embedding model. The exceptional combo of DWT/IWT change into is performed on the scrambled secret image and cover image to finish high protection and robustness. Hybrid turn into mixture method and case evaluation offered the various hiding environment. Experimental results and case be trained to supply the stego-image with perceptual invisibility, high safety and particular robustness. 
Mr. Madhusudhan Mishra et. al., [16] This paper introduces a brand new steganographic manner of secret information communication situated on Public Key Digital image steganography through combining public key cryptography with Digital image Steganography. The proposed scheme employs an RSA algorithm with 1024 bits key dimension for secret information encryption earlier than inserting it into cover picture and F5 steganographic manner to hide the encrypted message inside the cover picture imperceptibly. The F5 algorithm embeds the message into randomly chosen DCT coefficients. By employing matrix embedding which minimizes the changes to be made to the length of targeted message, it supplies high Steganographic ability, turbo speed and might avert visible and statistical assaults. The encryption key utilized in message encryption is enormous expansive to thwart known cryptanalytic attack. Experiments recommend that the stego image and cover pictures are perceptually similar. Further, the stego picture is effectively in opposition to image processing distortions.

\section{Proposed Methodology}

We presented QR code image steganography for multiple image and text hiding using 3-DWT and improved RSA algorithms. In this algorithm, firstly, enter the message data and four random numbers. We hide multiple secret information image into cover image in low frequency band of image by modifying the singular parameters and produce a secure stego image. Let the size of the color cover image is MXN and the size of color secret image is NXN. Firstly, divide cover image and secret image into three planes. After that, take the red component of the cover image and take all components of the first secret image, then hide first secret image into red component of the cover image. Second secret image is embedded in the green component of the cover image and third image is embedded into the blue component of the cover image. This process improves the security of image. For the security purpose, encrypt message data using an improved RSA algorithm. It is one of the famous security mechanism which is rely on number theory concept. It provides better confidential, secure communication over the internet.Its protection is depending on the complexity in factoring very big numbers. Depending on this standard, the RSA encryption utilizes main factorization as the trapdoor for encryption. It uses public key encryption in which anyone usesa public key to encrypt the data and send over the network. It provides authentication and security over the network in order to provide the private key to decrypt the information therefore only indented receiver can decrypt the The knowledge. The RSA algorithm is used for both data encryption and digital signature.

Proposed Algorithm

\section{Encryption Algorithm}

\section{Input: Text Message, Random Numbers Output: Encrypted Message}

1. Enter the text message (M) input for encryption.

2. Select $\mathrm{p}, \mathrm{q}, \mathrm{a}$ and $\mathrm{b}$ are random number, $p \neq q, a \neq b$.

3. Determine the value of modulus of random numbers:

$n=p \times q \times a \times b$.

4. Find the Euler totient function using formula:

$\emptyset(n)=(p-1) \times(q-1) \times(a-1) \times(b-1)$

5. Select integer e whose $\operatorname{gcd}=(\varnothing(n), e=1) ; 1<\mathrm{e}$

6. Find the modular multiplicative inverse and it is represented as a $d$

$d=e^{-1}(\bmod (\varnothing(n)))$ 
7. Public key $\mathrm{PU}=\{\mathrm{e}, \mathrm{n}\}$.

8. Private Key $P R=\{d, n\}$.

9. Encrypt message using this eqn:

Cipher $=M^{e} \bmod (n)$

\section{Embedding Algorithm}

\section{Input: Cover Image and Secret Image}

\section{Output: Stego Image}

10. Select a color image as a cover image.

11. Select three secret watermark image.

12. Split cover image and all secret images into three planes: Red (R), Green (G) and Blue (B).

13. Perform 3-DWT on the cover image of the red component to decompose it into four non-overlapping multi-resolution coefficient sets r_LL2, r_LH2, r_HL2, r_HH2

14. Perform 3-DWT on the cover image of the green component to decompose it into four non-overlapping multi-resolution coefficient sets g_LL2, g_LH2, g_HL2, g_HH2

15. Perform 3-DWT on the cover image of the blue component to decompose it into four non-overlapping multi-resolution coefficient sets b_LL2, b_LH2, b_HL2, b_HH2

16. Apply 3-DWT on the first secret image of the red, green and blue component to decompose it into four non-overlapping multi-resolution coefficient sets sr_LL2, sr_LH2, sr_HL2, sr_HH2, sg_LL2, sg_LH2, sg_HL2, sg_HH2 and sb_LL2, sb_LH2, sb_HL2, sb_HH2.

17. Apply 3-DWT on the second secret image of the red, green and blue component to decompose it into four non-overlapping multi-resolution coefficient sets sr_LL13, sr_LH13, sr_HL13, sr_HH13, sg_LL13, sg_LH13, sg_HL13, sg_HH13 and sb_LL13, sb_LH13, sb_HL13, sb_HH13.

18. Perform 3-DWT on the third secret image of the red, green and blue component to decompose it into four non-overlapping multi-resolution coefficient sets sr_LL23, sr_LH23, sr_HL23, sr_HH23, sg_LL23, sg_LH23, sg_HL23, sg_HH23 and sb_LL23, sb_LH23, sb_HL23, sb_HH23.

19. Hide secret image into cover image using below formula:

$$
\text { hide }=\text { Cover }_{\text {img }}+\gamma \times \text { Secret_img }
$$

where $\gamma$ is weight factor which is used to control the strength of an image and vary from

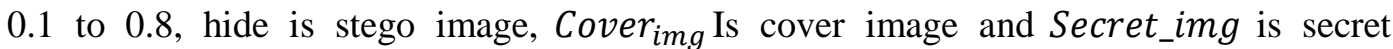
information.

20. Apply 3-inverse DWT on Stego image for reconstructing the matrix.

21. Calculate LSB of every pixel of embedded images.

22. Perform LSB on the embedded image to embed text into the embedded image.

$$
L S B=\bmod (\operatorname{hide}(i, j), 2)
$$

Where LSB is the Least Significant Bit, I is Encrypted image and $i, j$ is size of the image

\section{Extraction Algorithm}

Input: Stego Image

Output: Recover Three Secret Image 
23. Perform 3-DWT on the Stego image of the red component to decompose it into four non-overlapping multi-resolution coefficient sets exm_LL3,exm_LH3,exm_HL3,exm_HH3.

24. Apply 3-DWT on the Stego image of the green component to decompose it into four non-overlapping multi-resolution coefficient sets exm_LL13,exm_LH13,exm_HL13,exm_HH13

25. Perform 3-DWT on the Stego image of the blue component to decompose it into four non-overlapping multi-resolution coefficient sets exm_LL23,exm_LH23,exm_HL23,exm_HH23

26. Using this formula extract secret information from stego image:

Ext $_{\text {secret }}=\frac{\text { hide }- \text { Cover }_{\text {img }}}{\gamma}$

Where Ext_secret is recovered secret image

Decryption Algorithm

Input: Encrypted Message

Output: Decrypted Message

27. Recovered Original Message using below formula:

$M=C^{d} \bmod (n)$

28. Calculate Peak Signal Noise Ratio (PSNR) and Mean Square Error (MSE) value of stego and cover image.

$\operatorname{MSE}(x)=\frac{1}{N}\left\|x-x^{\wedge}\right\|^{2}=\frac{1}{N} \sum_{i=1}^{N}(x-x)^{2}$

29. Where $\mathrm{x}$ is cover image, $\mathrm{x}^{\wedge}$ is stego image, $\mathrm{N}$ is the size of the cover image

30. $\operatorname{PSNR}(x)=\frac{\left.10 X \log \left((\operatorname{double}(m))^{\wedge} 2\right)\right)}{\operatorname{MSE}(x)}$

Where $m$ is the maximum value of the cover image

31. Calculate StructuralSimilarity matrix (SSIM) of a cover image and secret image.

$S S I M=\frac{\operatorname{sum}(\text { sum }(\text { origImg } . * \text { origImg) }}{\text { sum(sum(distImg } . * \text { distImg }) \text { ) }}$

whereorigImg is a coverimage, distImg is recovered secret image 


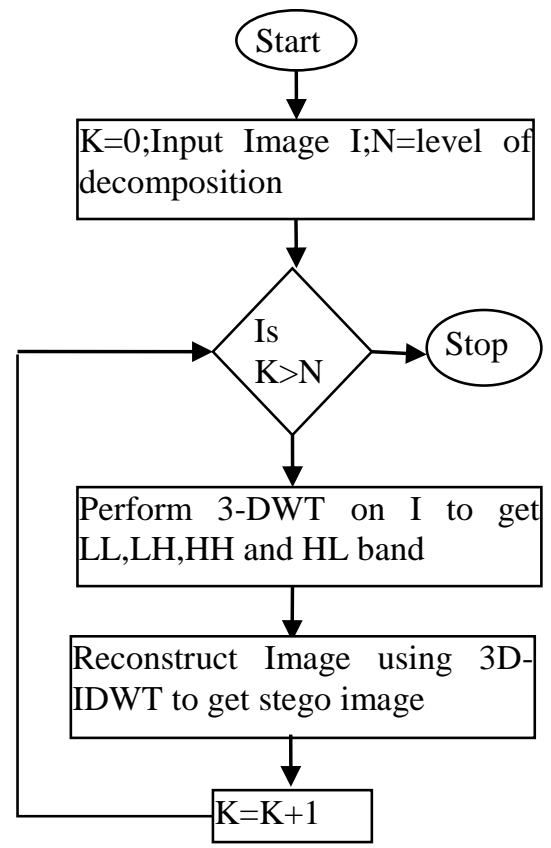

Figure 2. Flow Chart of Embedding Algorithm

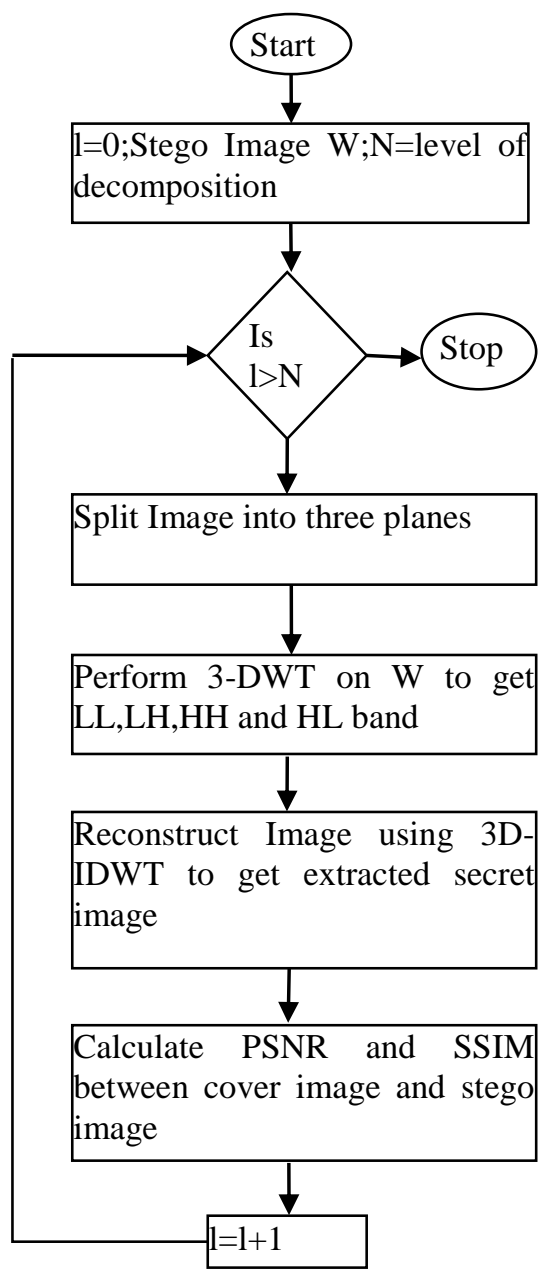

Figure 3. Flow Chart of Extraction Algorithm 


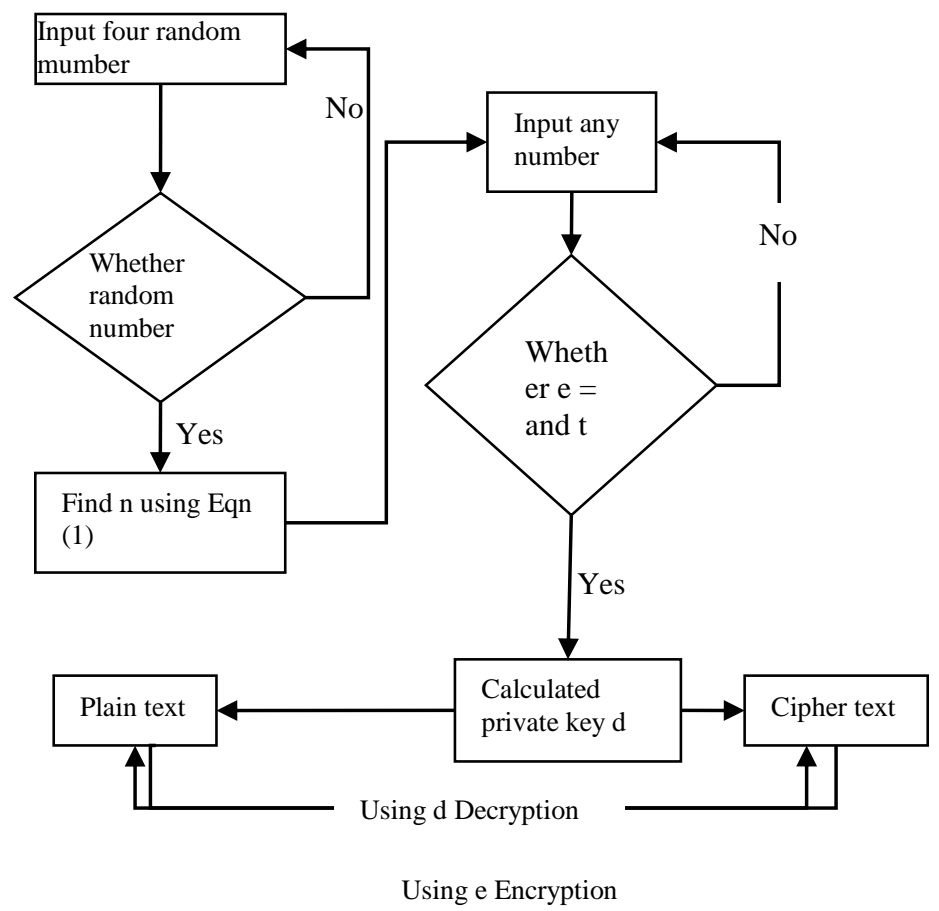

Figure 4. Flow Chart of Encryption and Decryption Algorithm

In Figure 2, initialize the value of $\mathrm{k}$ is zero, level of decomposition is $\mathrm{N}$, then perform four level DWT on both cover image and watermark image to get low frequency and high frequency band coefficients. Finally, apply inverse DWT to produce a watermarked image after embedding process.

In Figure 3, apply attacks on watermarked image, initialize the value of 1 is zero, level of decomposition is $\mathrm{N}$, then perform four level DWTon watermarked image to get low frequency and high frequency band coefficients. Finally, apply inverse DWTto produce an extracted watermark image after the extraction process.

In Figure 4, apply cryptography concepts to upturn security of the encrypted cloud data in the cloud servers with minimum consumption of cost and time at both decryption and encryptionprocesses.

\section{Performance Evaluation}

In this work, the experimental analysis is performed in MATLAB using an Image Processing toolbox. The resultant output is performed on eight images with varying weights. The performance measure is evaluated using PSNR and SSIM.

The simulation results suggest that this techniquemaintains good image quality. It is robust in comparison with different image processing operations. Figure 6 . Shows the original color cover image. Figure 7. And Figure 8. Shows the secret images and the extracted secret images respectively.

Compute the PSNR and MSE value of stegoand cover image.

$\operatorname{MSE}(x)=\frac{1}{N}\left\|x-x^{\wedge}\right\|^{2}=\frac{1}{N} \sum_{i=1}^{N}(x-x)^{2}$

Where $\mathrm{x}$ is cover image, $\mathrm{x}^{\wedge}$ is stego image, $\mathrm{N}$ is the size of thecover image

$\operatorname{PSNR}(x)=\frac{10 X \log \left(\left(\text { double }(m) \wedge^{\wedge} 2\right)\right)}{M S E(x)}$

Where $m$ is the maximum value of the cover image 
Calculate StructuralSimilarity matrix (SSIM) of a cover image and secret image.

$S S I M=\frac{\operatorname{sum}(\text { sum }(\text { origImg } . * \text { origImg })}{\text { sum(sum(distImg } * \text { distImg }) \text { ) }}$

WhereorigImgis a coverimage, distImg is recovered secret image

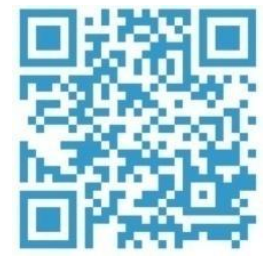

(a)

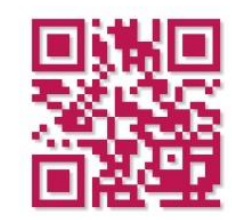

(b)

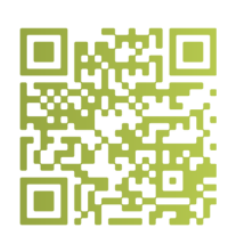

(c)

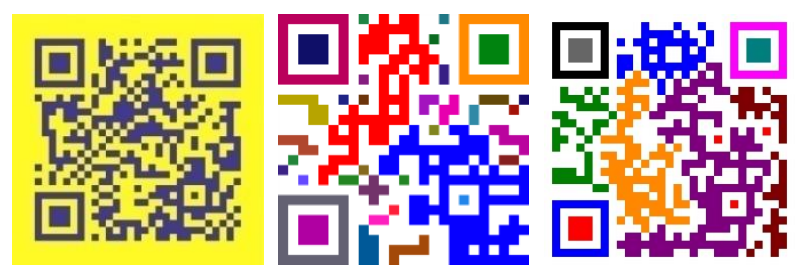

(d)

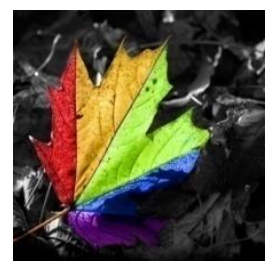

(e)

(f)

(g)

(h)

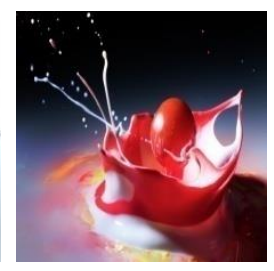

(i)

Figure 5. Experimental Dataset 


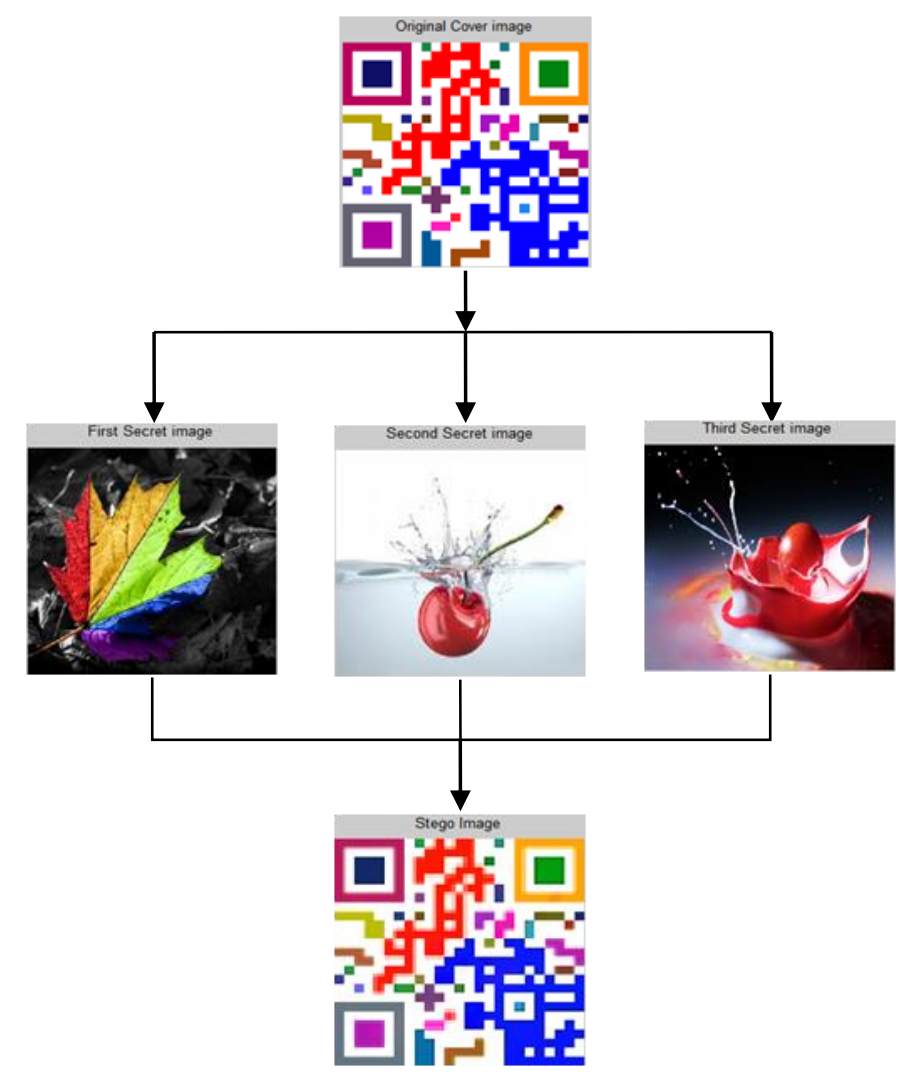

Figure 6. Top: Cover Image, Middle: Secret Image, Bottom: Stego Image

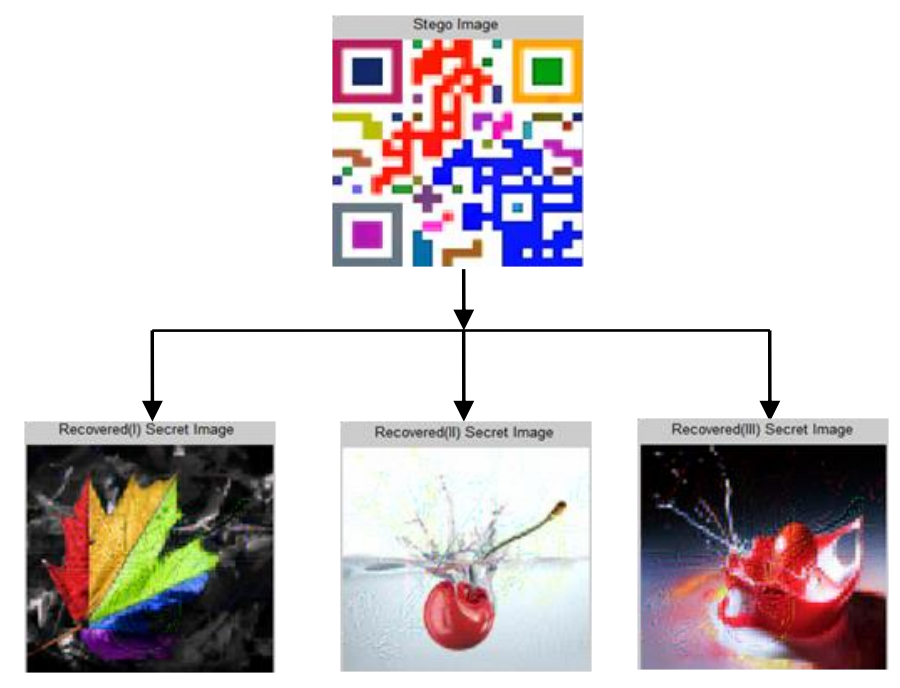

Figure 7. Top: Stego Image, Middle: Recovered Image 


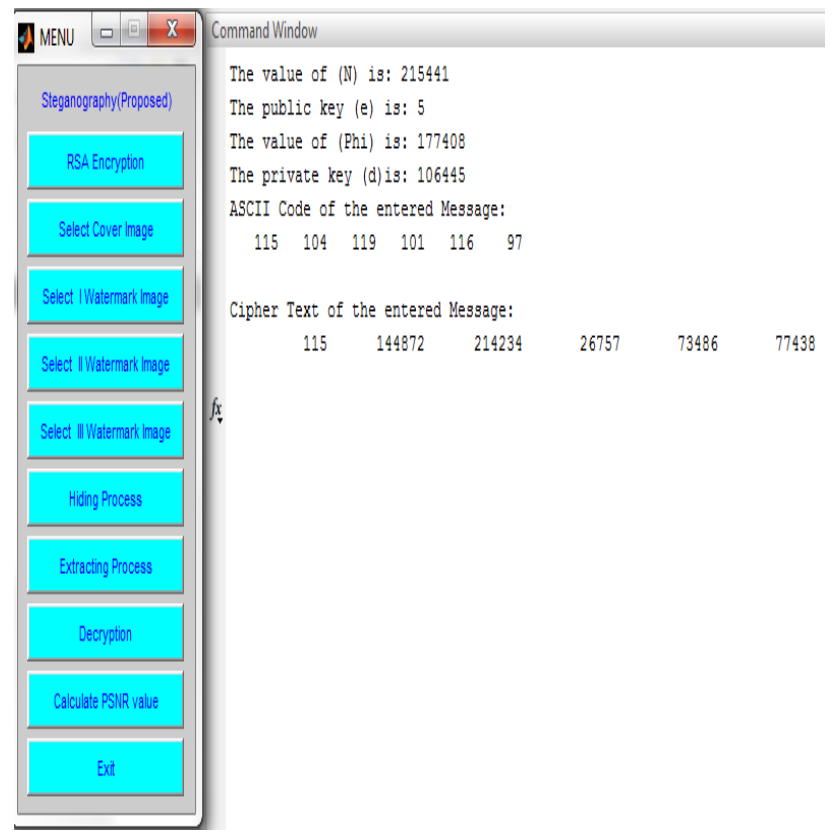

Figure 8. Encryption Process

Table 1. PSNR and MSE Comparison between Previous and Proposed System

\begin{tabular}{|c|c|c|c|c|}
\hline $\begin{array}{l}\text { Cover } \\
\text { Image }\end{array}$ & $\begin{array}{c}\text { Previous } \\
\text { Approach } \\
\text { PSNR }\end{array}$ & $\begin{array}{c}\text { Proposed } \\
\text { Approach } \\
\text { PSNR }\end{array}$ & $\begin{array}{c}\text { Previous } \\
\text { Approach } \\
\text { SSIM }\end{array}$ & $\begin{array}{c}\text { Pro } \\
\text { pose } \\
\mathrm{d} \\
\text { App } \\
\text { roac } \\
\mathrm{h} \\
\mathrm{SSI} \\
\mathrm{M}\end{array}$ \\
\hline (a) & 35.5995 & 80.7181 & 0.8902 & $\begin{array}{c}0.88 \\
95\end{array}$ \\
\hline (b) & 35.4825 & 84.0480 & 0.8989 & $\begin{array}{c}0.92 \\
30\end{array}$ \\
\hline (c) & 35.8511 & 89.2747 & 0.8987 & $\begin{array}{c}0.91 \\
78\end{array}$ \\
\hline (d) & 35.6589 & 80.6569 & 0.8547 & $\begin{array}{c}0.84 \\
98\end{array}$ \\
\hline (e) & 35.1623 & 83.5519 & 0.8912 & $\begin{array}{c}0.91 \\
69\end{array}$ \\
\hline (f) & 35.6604 & 84.9923 & 0.8967 & $\begin{array}{c}0.91 \\
73\end{array}$ \\
\hline
\end{tabular}

Table 2. SSIM Comparison between Previous and Proposed System with Varying Weight

\begin{tabular}{|c|c|c|}
\hline Weight & $\begin{array}{c}\text { Previous } \\
\text { Cover } \\
\text { Image } \\
(\mathrm{e})\end{array}$ & $\begin{array}{c}\text { Proposed } \\
\text { Cover } \\
\text { Image } \\
(\mathrm{e})\end{array}$ \\
\hline 0.1 & 0.8912 & 0.9169 \\
\hline 0.2 & 0.7971 & 0.8356 \\
\hline 0.3 & 0.7169 & 0.7663 \\
\hline 0.4 & 0.6481 & 0.7066 \\
\hline
\end{tabular}




\begin{tabular}{|l|l|l|}
\hline 0.5 & 0.5887 & 0.6547 \\
\hline 0.6 & 0.5370 & 0.6092 \\
\hline 0.7 & 0.4918 & 0.5689 \\
\hline 0.8 & 0.4520 & 0.5329 \\
\hline
\end{tabular}

Table 3. SSIM with Weight 0.1 of Proposed System

\begin{tabular}{|c|c|c|c|}
\hline Cover Image & $\begin{array}{c}\text { Secret } \\
\text { Image (g) }\end{array}$ & $\begin{array}{c}\text { Secret } \\
\text { Image (h) }\end{array}$ & $\begin{array}{c}\text { Secret } \\
\text { Image (i) }\end{array}$ \\
\hline (a) & 1.0211 & 0.9969 & 1.0041 \\
\hline (b) & 1.0211 & 0.9969 & 1.0041 \\
\hline (c) & 1.0211 & 0.9969 & 1.0041 \\
\hline$(\mathrm{d})$ & 1.0211 & 0.9969 & 1.0041 \\
\hline$(\mathrm{e})$ & 1.0211 & 0.9969 & 1.0041 \\
\hline (f) & 1.0211 & 0.9969 & 1.0041 \\
\hline
\end{tabular}

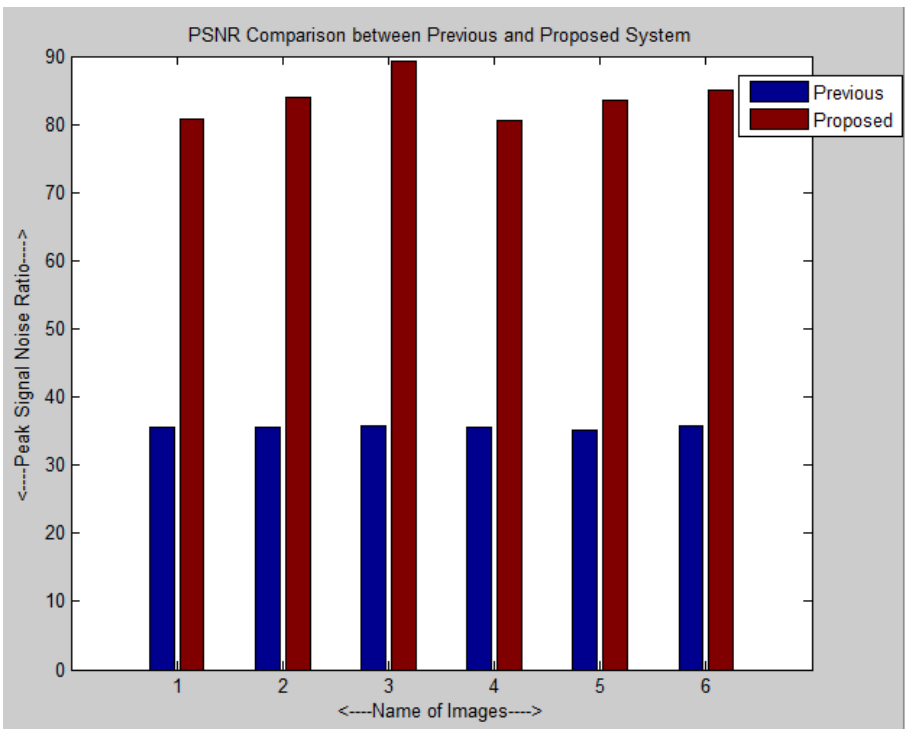

\section{Graph1. PSNR Comparison between Previous and Proposed System}

\section{Conclusion}

This researchpresents QR code image steganography For multiple image and text hiding using 3-DWT and improved RSA for security mechanism. The trial results, evaluate the eventual outcome of the existing technique and proposed technique considering the parameters of PSNR and SSIM. From the results we can say that the proposed technique works better than the previous technique. The PSNR is $84 \%$ after the embedding process. SSIM is close to 1 which shows the robustness to the attacks. The performance analysis validates that the presented steganography method has good imperceptibility too. The comparison outcomes with DWT based algorithm. It shows that the superiority of our method. In the future scope, enhance the extraction process for image quality. Also apply attacks on stego image and recovered secret image. 


\section{References}

[1] P. Patel and Y. Patel, "Secure and authentic DCT image steganography through DWT - SVD based Digital watermarking with RSA encryption", Fifth International Conference on Communication Systems and Network Technologies, IEEE, (2015).

[2] G. J. Simmons, "The Prisoners' Problem and the Subliminal Channel”, Proceedings of CRYPTO, vol. 83, (1984), pp. 51-57.

[3] R. J. Anderson, "Stretching the Limits of Steganography, Information Hiding, Springer Lecture Notes in Computer Science", vol. 1174, (1996), pp. 39-48.

[4] S. Craver, "On Public-key Steganography in the Presence of an Active Warden", Proceedings of 2nd International Workshop on Information Hiding., (1998), pp. 355-368.

[5] R. J. Anderson and F. A.P. Petitcolas, "On the limits of steganography", IEEE Journal on Selected Areas in Communications (J-SAC), Special Issue on Copyright and Privacy Protection, vol. 16, (1998), pp. 474- 481

[6] N. F. Johnson and S. Jajodia, "Steganography: seeing the unseen", in IEEE Computer, vol. 16, (1998), pp. 26-34.

[7] T. Mrkel., JHP Eloff and MS Olivier, "An Overview of Image Steganography", in Proceedings of the fifth annual Information Security South Africa Conference, (2005).

[8] S. Bhattacharyya and G. Sanyal, "A Robust Image Steganography using DWT Difference Modulation (DWTDM)”, I. J. Computer Network and Information Security, vol. 7, (2012), pp. 27-40.

[9] K. Naresh and P. N. Pillai, "QR Verification System Using RSA Algorithm”, ijisr, (2014).

[10] S. V. Patel, S. V. Patel (2011), "Invisible Digital Video Watermarking Using 4-level DWT", National Conference on Recent Trends in Engineering \& Technology, B.V.M. Engineering College, V.V. Nagar, Gujarat, India, (2011) May 13-14.

[11] Ms. A. Gupta and Er. R. kaur, "A Study of Video Watermarking Techniques Based on Energy Model", IRJET, (2015).

[12] V. Kumar and D. Kumar, "Performance evaluation of DWT based image steganography", Advance Computing Conference (IACC), 2010 IEEE 2nd International, vol., no., (2010), Feb. 19-20, pp. 223,228.

[13] A. Nag, S. Biswas, D. Sarkar and P. P. Sarkar, "A Novel Technique for Image Steganography Based on DWT and Huffman Encoding”, International Journal of Computer Science and Security, (IJCSS), vol. 4, Issue (6).

[14] R. J. Jaiswal, R. Soni and P. Mahale, "Reformed RSA algorithm based on Prime Number", NCETIT, (2014).

[15] P. Ganesan and R. Bhavani, “A high secure and robust Image steganography using dual wavelet blending model", Journal of Computer Science, vol. 9, no. 3, (2013), pp. 277-284.

[16] Mr. M. Mishra, Mr. G. Tiwari and Mr. A. K. Yadav, "Secret Communication using Public 1(ey Steganography”, IEEE, (2014). 